\title{
Comprehensive Automated Thin-Section Characterization Combined with Quantitative Major-Trace Element Analysis on a Single SEM
}

Rosie Jones $^{1}$, Matt Hiscock ${ }^{2}$, Pat Trimby ${ }^{3}$, Robyn Gardner ${ }^{4}$, Richard Mclaughlin ${ }^{5}$ and Simon Burgess ${ }^{6}$

${ }^{1}$ Oxford Instruments NanoAnalysis, High Wycombe, United Kingdom, ${ }^{2}$ Oxford Instruments NanoAnalysis, United States, ${ }^{3}$ Oxford Instruments, High Wycombe, England, United Kingdom, ${ }^{4}$ Macquarie University, Australia, ${ }^{5}$ Oxford Instruments NanoAnalysis, Concord, United States, ${ }^{6}$ Oxford Instruments NanoAnalysis, High Wycombe, England, United Kingdom

An analytically equipped scanning electron microscope (SEM) is an exceptionally powerful tool, able to bring together information from multiple techniques to enable in-depth characterization of materials. We usually have a specific set of analytical requirements for geological samples that can be addressed by a combination of in-situ approaches in a SEM. These requirements often lead to varying levels and types of information being obtained from different parts of a thin section. There is frequently a need to understand how mineral composition/abundance varies over the scale of a thin section and the specific chemistry of the minerals present (including high sensitivity measurements of trace element concentrations/compositional variations). It may also be desirable to know the textural/crystalline properties of the rock to make links between what is observed compositionally and the physical processes that have affected it during its history. These requirements often need analyses covering large proportions of the sample(s), so that a statistically representative dataset can be generated.

In this submission, we investigate a gabbro sample and demonstrate a workflow that allows a thin section to be comprehensively characterized on a single, SEM. We consider the automated acquisition of data from large areas of the sample, including an assessment of the bulk mineralogy via Energy Dispersive Xray Spectrometry (EDS) based automated approaches, and a characterization of grain textures by Electron Backscatter Diffraction (EBSD). We discuss the different automated approaches available, their applicability, and advantages over more manual forms of analysis. We also consider how Wavelength Dispersive X-ray Spectrometry (WDS) on a SEM can provide high quality minor/trace element analysis in conjunction with EDS (major elements) and provide results with a similar level of accuracy and precision as a dedicated electron microprobe (i.e., EPMA).

The analysis shown here begins with a large area EDS map for an overview of the sample (Fig. 1a). In this case, the map shown is $33.1 \times 17.8 \mathrm{~mm}$ with a pixel size of c. $3.5 \mu \mathrm{m}$ and was acquired over a period of 7 hours with no human input beyond an initial 5-minute setup. Within the map (1161 images stitched into a single montage), it is possible to see the large-scale structure of the gabbro and the chemical variations due to the minerals present. It is then possible to extract, from the original map dataset (without any further acquisition), a particle/grain analysis (Fig. 1b). This process gathers morphological information from each of the grains that make up the sample and combines it with compositional data extracted from the EDS map. This grain-by-grain data is then classified to provide information on the minerals present and their relative proportions. As the data is extracted automatically from the whole sample, it is acquired without bias, at higher spatial resolution, and far less laboriously than equivalent optical analysis. These advantages are of course in addition to the direct compositional measurements, which could not be made optically. This aids with mineral identification and highlights features such as chemical zonation and alteration. 
In certain situations, where there is already a good overview understanding of the sample, it may not be necessary to perform the initial EDS mapping step described here, and instead proceed directly to the automated feature analysis. In such cases, the time to result is improved as automated feature analysis is significantly faster than mapping as one spectrum is acquired per feature rather than per pixel.

Automated large area EBSD analysis adds crystallographic measurements to our dataset. This allows phase identifications to be confirmed by the crystal structure and correlated against directly measured grain sizes - which would be very hard to measure individually - giving an in-depth understanding of the rock (Fig. 2).

Following the automated, large area acquisitions, individual mineral grains were identified for quantitative compositional analysis using both WDS, and combined EDS-WDS. Mineral compositions have previously been determined for this sample by electron microprobe [1]. One of the aims of this study is to compare the results of SEM-based EDS/WDS analysis, with previously acquired EPMA data, by collecting data from the same locations on the thin section using similar analytical conditions. The sample was analyzed using a Wave WDS spectrometer and an Ultim Max $170 \mathrm{~mm}^{2}$ EDS detector in the AZtecWave software. An accelerating voltage of $15 \mathrm{kV}$ and a beam current of $25 \mathrm{nA}$ were used, and WDS peak counting times varied between 10 and $40 \mathrm{~s}$. Good agreement is observed between the compositional data obtained via EPMA and SEM-based EDS/WDS. For example, the table below shows the average results obtained for olivine on the same 8 sample points using the different techniques. In the SEM EDS+WDS case, the major elements ( $\mathrm{Si}, \mathrm{Fe}, \mathrm{Mg}$ ) were analyzed by EDS, and the minor-trace elements by WDS. In all cases the $\mathrm{O}$ is calculated by stoichiometry and the analytical totals are unnormalized. Both the major and minor element concentrations obtained using the different techniques are within error.

\begin{tabular}{lllllllll} 
& \multicolumn{2}{l}{ Element $(\mathbf{w t} . \%)$} & & & & \\
& $\mathbf{S i}$ & $\mathbf{F e}$ & $\mathbf{N i}$ & $\mathbf{M n}$ & $\mathbf{M g}$ & $\mathbf{C a}$ & $\mathbf{O}$ & Total \\
Electron microprobe average & 16.87 & 25.90 & 0.03 & 0.42 & 18.25 & 0.02 & 38.79 & 100.28 \\
$l \sigma$ & 0.085 & 0.396 & 0.011 & 0.021 & 0.211 & 0.008 & 0.172 & 0.474 \\
SEM WDS-only average & 16.79 & 25.39 & 0.04 & 0.43 & 18.56 & 0.01 & 38.75 & 99.97 \\
$1 \sigma$ & 0.327 & 0.309 & 0.014 & 0.027 & 0.310 & 0.004 & 0.268 & 0.487 \\
SEM EDS+WDS average & 17.02 & 25.24 & 0.04 & 0.43 & 18.14 & 0.01 & 38.69 & 99.57 \\
$l \sigma$ & 0.059 & 0.320 & 0.014 & 0.027 & 0.204 & 0.004 & 0.118 & 0.201
\end{tabular}

This study demonstrates the ease and speed at which geological samples, and other materials, can be comprehensively characterized on an analytical SEM using a combination of automated, large area mapping/feature analysis, followed by high quality, quantitative major-trace element analysis on individual points of interest. 

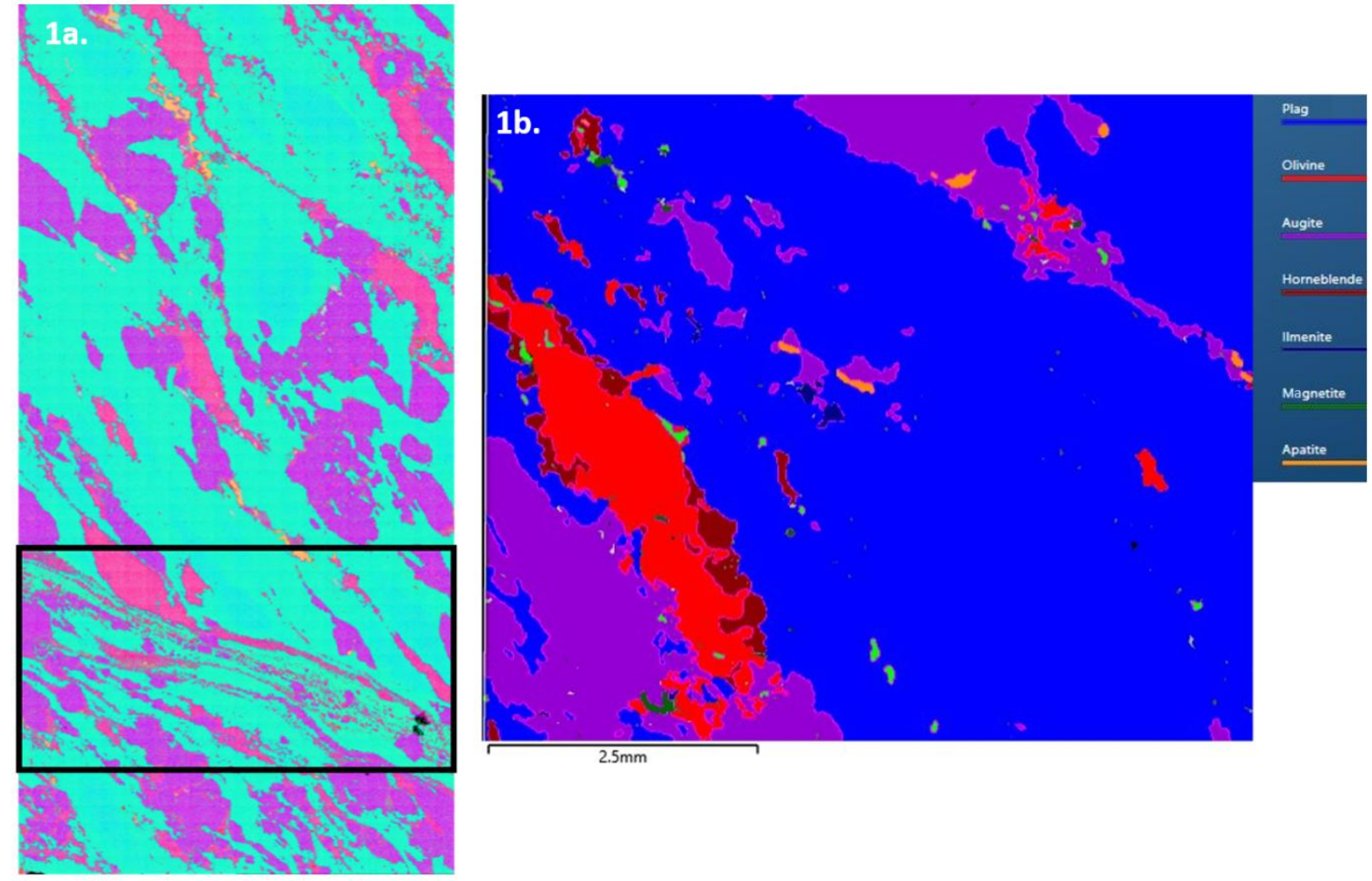

\section{P Ti Fe Mg Na Ca}

Figure 1. (a) Large area EDS map of selected elements showing the large scale distribution of elements that make up the sample. The black box shows where the EBSD map, shown in Figure 2, was acquired from. (b) Automated feature analysis of the gabbro sample, showing the classification of multiple mineral phases.

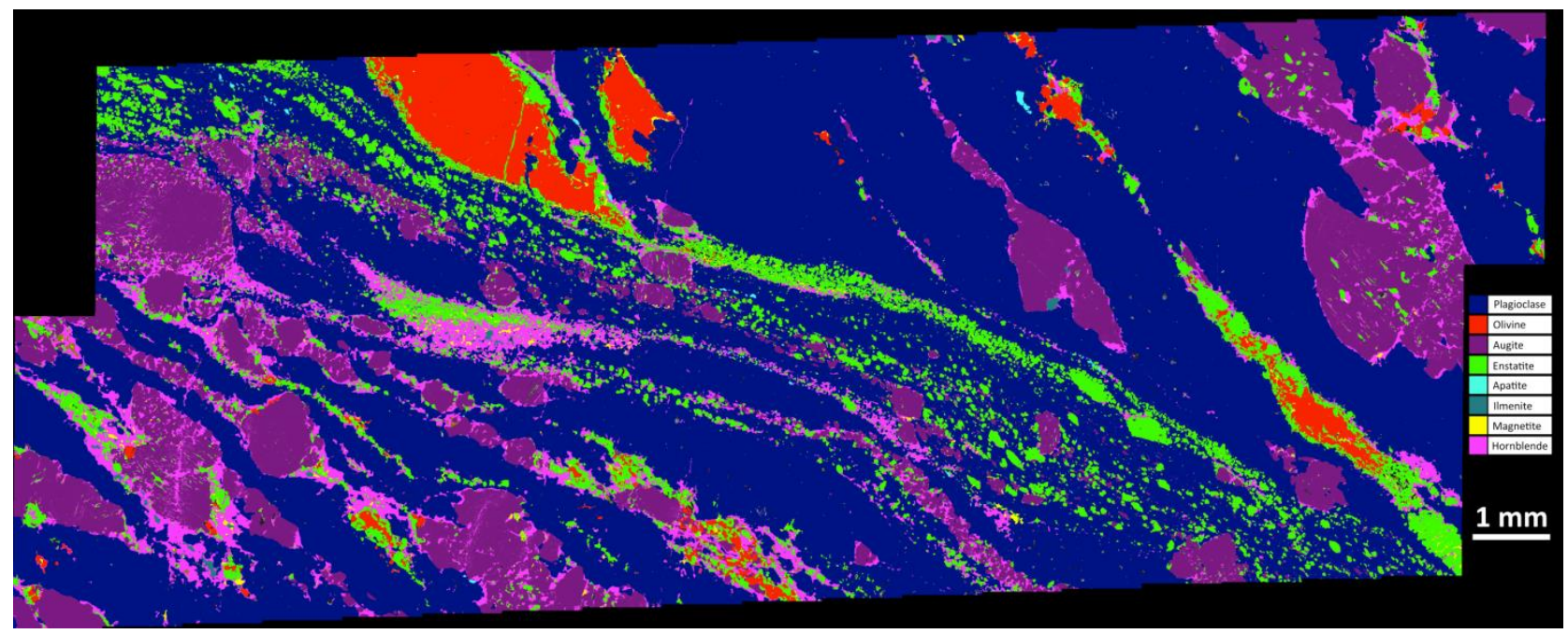

Figure 2. An EBSD phase map from a high-resolution montage across a shear zone located just below the center of the thin section shown in Figure 1a.

\section{References}

[1] Gardner, R.L., Piazolo, S., Daczko, N.R. and Trimby, P., 2020. Microstructures reveal multistage melt present strain localisation in mid-ocean gabbros. Lithos, 366, p.105572. 